\title{
Biologia de aves capturadas em um fragmento de Mata Atlântica, Igarassu, Pernambuco, Brasil
}

\author{
Vivyanne S. Magalhães 1; Severino M. de Azevedo Júnior 1; Rachel M. de Lyra-Neves 2; \\ Wallace R. Telino-Júnior ${ }^{2} \&$ Daniela P. de Souza ${ }^{1}$
}

\author{
1 Programa de Pós-Graduação em Biologia Animal, Departamento de Zoologia, Universidade Federal de Pernambuco. \\ Avenida Professor Moraes Rego 1235, Cidade Universitária, 50670-420 Recife, Pernambuco, Brasil. \\ E-mail: vivyannes@gmail.com; smaj@ufpe.br \\ 2 Universidade Federal Rural de Pernambuco, Unidade Acadêmica de Garanhuns. Avenida Bom Pastor, Boa Vista, \\ 55296-901, Garanhuns, Pernambuco,Brasil. E-mail: telinojr@uag.ufrpe.br; rmlneves@uag.ufrpe.br
}

\begin{abstract}
Biology of birds captured in an Atlantic Forest fragment at Igarassu, Pernambuco, Brazil. We carried out a study about the biology of the avifauna of Refúgio Ecológico Charles Darwin, a 60 ha fragment of Atlantic Forest in the town of Igarassu, Pernambuco. To obtain information about species of this bioma, observations were done between August, 1996 and July, 1997 and monthly captures using mist nets were conducted between July, 2003 and June, 2004. Among observations, captures, recaptures and recovers, 151 species (31 families) were registered in the study area, and 456 birds (from 53 species and 25 families) were trapped in the nets. Ten species had been recovered (life span from six to eight years). The amount of captures was greater during months with higher temperatures. The majority of captured species (52,8\%) had frequency of occurrence less than $25 \%$; Manacus manacus (Linnaeus, 1766), Arremon taciturnus (Hermann, 1783), Neopelma pallescens (Lafresnaye, 1853), and Turdus leucomelas Vieillot, 1818 were the most frequent. We observed significant correlation between average body mass and sex; length of the wing and tarsus and diameter of tarsus with sex, and of molt with seasons. The main period with molt associated with the brood patches was from March to May (with a peak in May). The findings reinforce the unpredictability of the effects of alterations in the structure of bird community in long term. The population unbalance can increase the possibilities of extinction, highlighting the need of new alternatives for the protection of biodiversity, specially in forest fragments.
\end{abstract}

KEY WORDS. Bird community; biometry; capture; conservation; fragmentation; molt.

RESUMO. Foram estudados alguns aspectos da biologia da avifauna do Refúgio Ecológico Charles Darwin, fragmento de 60 ha de Mata Atlântica, no município de Igarassu, Pernambuco. Objetivando obter informações acerca das espécies desse bioma, foram realizadas observações entre agosto de 1996 e julho de 1997 e capturas mensais utilizando redes de neblina, entre julho de 2003 e junho de 2004. Entre observações, capturas, recapturas e recuperações, foram registradas 151 espécies (31 famílias) para a área, onde 456 aves (53 espécies/ 25 famílias) foram capturadas com redes ornitológicas. Foram recuperadas 10 espécies (tempo de anilha de seis a oito anos). $\mathrm{O}$ número de capturas foi maior nos meses mais quentes. A maioria das espécies capturadas (52,8\%) teve freqüência de ocorrência menor que $25 \%$, sendo Manacus manacus (Linnaeus, 1766), Arremon taciturnus (Hermann, 1783), Neopelma pallescens (Lafresnaye, 1853) e Turdus leucomelas Vieillot, 1818 as mais freqüentes. Houve correlação significativa entre as análises dos valores médios entre massa corpórea e sexo, dados biométricos (medidas da asa, tarso e diâmetro do tarso) e sexo e entre mudas e estação do ano. O maior período com muda associada à placa de incubação foi de março a maio (pico em maio). Os resultados fortaleceram a imprevisibilidade dos efeitos das alterações ambientais na estrutura da comunidade de aves em longo prazo. Reforçam ainda que os desequilíbrios populacionais possam vir a aumentar as chances de extinção, sendo necessárias novas alternativas para a proteção da biodiversidade, sobretudo em fragmentos florestais.

PALAVRAS-CHAVE. Avifauna; biometria; conservação; captura; fragmentação; mudas.

A rapidez da destruição atual dos ambientes naturais não tem precedente na história da floresta tropical úmida (BierRegaArd et al. 1992). Neste contexto, a Mata Atlântica é um dos ecossistemas florestais mais ameaçados e devastados pelo homem (Fonseca 1985, Ranta et al. 1998, Myers et al. 2000), restando apenas 7,3\% da sua área original (Івама 2003).

Revista Brasileira de Zoologia 24 (4): 950-964, dezembro 2007 
No início do século XX, a região nordeste possuía 36,8\% de sua área coberta com este ecossistema e o Estado de Pernambuco 34,14\% (GonZAgA DE CAmpos 1912). Atualmente o Estado mantém apenas $2 \%$ da cobertura original, em fragmentos rodeados por monoculturas e aglomerados urbanos (VIANa et al. 1997, Chiarello 1999, Silva \& Tabarelli 2000), sendo 48\% dos remanescentes abaixo de 10 ha e apenas 7\% acima de 100 ha (média de 34 ha) (RANTA et al. 1998).

A destruição da floresta leva a eliminação de muitas populações e a diminuição da diversidade genética de espécies (Brown \& Brown 1992, Morellato \& Haddad 2000), iniciando um processo contínuo de extinção local, onde a composição e a diversidade biológica da comunidade passam por transição até o equilíbrio (TABARELli 2000). Willis (1979) e BierREgAard et al. (1992) relacionaram diminuição da área florestal com decréscimo no número de espécies de aves, por isso entende-se que a presença ou ausência de certas espécies determina a situação ecológica de ecossistemas específicos (TELINo-Júnior et al. 2000).

Para Laurance (1991) e TABarelli (2000), a fragmentação apresenta efeitos em longo prazo sobre as populações por alterar os principais processos ecológicos: polinização, predação, comportamento territorial e hábitos alimentares. Os distúrbios antropogênicos no hábitat natural, geradores de tais alterações, levam a necessidade de monitorar o tamanho e qualidade das populações, o que pode ser feito através de métodos simples, como o índice para monitoramento do grau de conservação de populações de aves em fragmentos da Mata Atlântica (ANCIÃEs \& MARINI 2000a, b).

Segundo Azevedo Júnior et al. (2001), diversos estudos já demonstraram a importância da marcação de aves, sobretudo aqueles sobre biologia, longevidade, deslocamentos e migrações. Sıck (1997) afirma que o anilhamento é útil, se não indispensável, no estudo de populações locais, incluindo as que não migram.

Embora as aves, de um modo geral, sejam um grupo amplamente estudado, informações relativas à bionomia e biometria são escassas (Foster 1975, Clark JúNIOR 1979, REINERT et al. 1996). Estudos prolongados sobre mudas de penas de aves neotropicais são raros (MARINI \& DURãEs 2001). Os autores que fazem inferência acerca da massa corpórea e de dados biométricos, em geral apresentam informações pontuais, como Baldwin \& Kendeigh (1938), McNeil (1971), Clark Júnior (1979), ONiki (1981), Oniki \& Willis (1993), ReInert et al. (1996). Poucas são as pesquisas que mostram como as espécies respondem ao atual estado de fragmentação na região nordestina. Para a área estudada, trabalhos anteriores registraram espécies endêmicas e ameaçadas de extinção, através de pesquisas com anilhamento e estudos da biologia de aves entre 1996 e 1997 (Lyra-Neves et al. 2000, Telino-Júnior et al. 2000).

Este trabalho visa classificar a avifauna local quanto ao endemismo e ameaça de extinção através de capturas e recapturas e verificar a existência de correlação entre caracte- rísticas fenotípicas dos indivíduos de uma mesma espécie e variáveis abióticas. Com isso, espera-se subsidiar a elaboração de estratégias de conservação da avifauna e dos seus habitats, sobretudo aqueles em declínio, como a Floresta Atlântica.

\section{MATERIAL E MÉTODOS}

O estudo se deu numa propriedade privada de 60 ha, (Refúgio Ecológico Charles Darwin - RECD) na Zona da Mata Úmida Norte de Pernambuco (Andrade-Lima 1960, Lyra-Neves et al. 2000) (Figs 1-3). O RECD está situado no Município de Igarassu (entre $7^{\circ} 48^{\prime}-7^{\circ} 49^{\prime} \mathrm{S}$ e $34^{\circ} 56^{\prime}-34^{\circ} 57^{\prime} \mathrm{W}$ ), distando $34 \mathrm{~km}$ da cidade de Recife, Pernambuco e compõe o Complexo Litorâneo da Reserva da Biosfera da Mata Atlântica. Foi criado há cerca de 40 anos e hoje funciona como Centro de Pesquisas (Lima 1998).

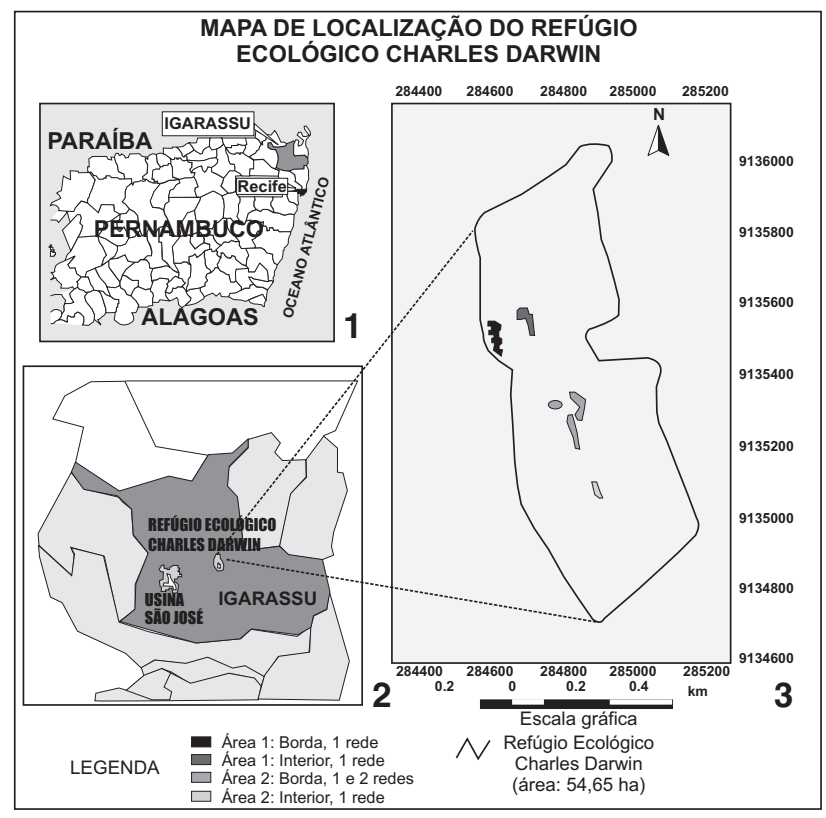

Figuras 1-3. Planta Diretora de Igarassu (Escala 1:760.000): (1) localização do município de Igarassu; (2) posição geográfica do Refúgio Ecológico Charles Darwin (RECD); (3) localização georeferenciada das redes. Fonte: Sintaxe Consultoria.

Sua área é um remanescente de Mata Atlântica com formação secundária em recomposição, embora as propriedades vizinhas estejam desmatadas para agropecuária. A vegetação divide-se em capoeirão, capoeira rala, vegetação arbustiva e campos (FIDEM 1993).

O registro de espécies no RECD ocorreu em duas fases: entre agosto de 1996 e julho de 1997 por capturas (900 horas/ redes) e observações visuais e auditivas diretas (450 horas/observações) e de julho de 2003 a junho de 2004, por captura mensal, durante três dias. Foram utilizadas 12 redes de neblina 
(mist-net), com $36 \mathrm{~mm}$ de malha, $12 \mathrm{~m}$ de comprimento e cerca de $3 \mathrm{~m}$ de altura. As redes foram abertas entre 5 e 10 horas e 15 e 17 horas sendo revisadas a cada hora, num esforço amostral próximo a 490 horas/rede em 12 meses.

Os indivíduos foram identificados até espécie (DunNing 1987, Ridgely \& Tudor 1994a, b, Sick 1997, Souza 2002). A nomenclatura científica e vulgar utilizada foi proposta pelo Свво (2006). As aves capturadas receberam anilhas metálicas do Centro Nacional de Pesquisa para a Conservação de Aves Silvestres (CEMAVE/IBAMA).

Todas as informações (dados abióticos, biometria, idade, sexo, mudas, placa de incubação, guildas alimentares e sensibilidade a distúrbios) foram anotadas em planilha de campo. Foram consideradas recuperações as capturas de animais previamente anilhados por outros pesquisadores. Indivíduos recuperados que caíram mais de uma vez na rede, passaram a ser considerados recapturas.

Dados de temperatura e pluviometria de 2003 e 2004 foram obtidos junto a Seção de Observação e Meteorologia Aplicada (SEOMA), do Instituto Nacional de Meteorologia (INMET). Os índices pluviométricos, no período estudado, variaram entre $26,8 \mathrm{~mm}$ (novembro de 2003) e 538,9 $\mathrm{mm}$ (junho de 2004). A temperatura e a umidade relativa do ar tiveram médias de $26,1^{\circ} \mathrm{C}$ e $79,4 \%$, respectivamente. O período de estiagem concentrou-se entre agosto e dezembro de 2003.

Foi aferido comprimento das asas e cauda com régua metálica milimetrada e tarsos e diâmetro dos tarsos com paquímetro (precisão de 0,02 mm). A massa corpórea foi obtida com balanças Pesola ${ }^{\circledR}$ tipo dinamômetro (precisão de 1 e 2 g). A determinação da idade e sexo, quando possível, foi baseada na plumagem.

Quanto às mudas, foram observadas: penas de contorno, penas da asa (rêmiges) e penas da cauda (retrizes). Foi considerada muda de contorno quando havia penas em crescimento (canhão) na região da cabeça, dorso e ventre, num mínimo de três penas em quaisquer dessas regiões. Nas penas de vôo da asa, apenas as mudas das primárias foram observadas (Miller 1961, Avery 1985). Para a análise das mudas, as aves recapturadas ou recuperadas em diferentes meses foram tratadas como novos registros, mas somente no primeiro registro individual do mês amostrado (Marini \& Durães 2001).

Quanto ao estudo reprodutivo, foi analisada a presença de placa de incubação ou choco, seguindo a classificação adotada pelo CEMAVE (IвAma 1994).

As guildas alimentares foram agrupadas em cinco categorias: Onívoras (O), Granívoras (G), Insetívoras (I), Frugívoras (F) e Nectarívoras (N), seguindo Willis (1979), Terborgh et al. (1990), Sick (1997) e Sigrist (2006).

As espécies foram classificadas quanto à sensibilidade aos distúrbios, prioridade de conservação e prioridade de pesquisa ao ambiente alterado, segundo PARKER III et al. (1996). Aquelas endêmicas e ameaçadas de extinção foram definidas segundo a Lista Nacional das Espécies da Fauna Brasileira Ameaçadas de Extinção (IвAMA 2003).
Dos dados gerados foram obtidas distribuições absolutas e percentuais das variáveis nominais ou categóricas e média e desvio padrão das variáveis numéricas para análise dos dados de massa corpórea, inferência entre sexos, estação do ano e medidas biométricas dos caracteres fenotípicos.

A freqüência de ocorrência (FO) foi calculada pela fórmula "FO = A/n.100", onde "A" é o número de fases de campo com registro da espécie e " $\mathrm{n}$ " o total de amostragens.

Utilizou-se teste t-Student para verificar se houve diferença significativa para variáveis contínuas com variâncias iguais ou desiguais da massa corpórea entre sexos, da massa corpórea durante estação do ano (seca e chuvosa) e das medidas de asa, tarso e diâmetro do tarso entre sexos, para espécies com $n>5$ (excluídos não adultos, sexo indeterminado ou recapturas). A verificação da hipótese de igualdade de variâncias foi realizada através do teste $\mathrm{F}$.

O teste do Qui-quadrado de Pearson foi utilizado para verificar se houve diferença significativa na proporção de presença dos tipos de muda analisados (rêmiges, contorno e retriz) entre a estação chuvosa e seca ao longo do ano.

Para avaliar as semelhanças ecológicas das amostras, utilizou-se o Método de Ordenação (Análise de Componentes Principais - ACP) cruzando-se os dados de FO com fatores abióticos (temperatura e umidade). Foram destacadas as espécies com FO superior a 58\%, consideradas relevantes pela presença na maioria das amostras, com índice de correlação de Pearson, utilizando-se o Software NTSYS pc 2.1. Foram utilizadas médias mensais para temperatura e totais mensais para umidade, utilizando a matriz qualitativa (presença versus ausência).

Para os cálculos estatísticos, foi utilizado o programa SAS (Statistical Analysis System), versão 8, e o nível de significância foi de $5 \%(0,05)$.

\section{RESULTADOS}

Foram registradas 151 espécies de aves, de 31 famílias, considerando todos os períodos analisados. Entre 2003 e 2004, foram 456 indivíduos (53 espécies de 25 famílias) entre capturas (335), recapturas (98) e recuperações (23) (Tab. I). Dos 23 recuperados, $14(3,07 \%)$ dos indivíduos amostrados caíram na rede uma única vez, abrangendo 10 espécies de 5 famílias diferentes. Tais recuperações ocorreram seis a oito anos após a primeira captura.

A média de espécies presentes nos meses mais frios (julho a outubro de 2003; maio e junho de 2004 , entre $24,3^{\circ} \mathrm{C}$ e $26^{\circ} \mathrm{C}$ ), foi inferior (16 espécies) à média de 21 espécies, capturadas nos meses mais quentes (novembro de 2003 a abril de 2004 , entre $26,6^{\circ} \mathrm{C}$ e $28,1^{\circ} \mathrm{C}$ ) (INMET 2004). No período de estiagem, as capturas apresentaram alta, com o maior registro ( $\mathrm{n}=$ 63) para o mês de dezembro.

A maior parte $(52,8 \%)$ da avifauna capturada apresentou FO inferior a 25\%, (presente em menos de quatro do total de 12 visitas - Tab. II). Manacus manacus (Linnaeus, 1766) com 91,67\%, Arremon taciturnus (Hermann, 1783), Neopelma pallescens 
Tabela I. Listagem das espécies registradas no Refúgio Ecológico Charles Darwin, através de observações e captura em rede. (CP) Número de capturas, (RC) número de recapturas, (RP) número de recuperações, (SD) sensibilidade aos distúrbios, (PC) prioridade de conservação, (PP) prioridade de pesquisa, (A) alta, (B) baixa, (M) média.

\begin{tabular}{|c|c|c|c|c|c|c|c|c|}
\hline \multirow{2}{*}{ Espécies } & \multirow{2}{*}{ Nome popular } & \multicolumn{4}{|c|}{$2003-2004$} & \multirow{2}{*}{ SD } & \multirow{2}{*}{ PC } & \multirow{2}{*}{ PP } \\
\hline & & $\mathrm{CP}$ & $\mathrm{RC}$ & $\mathrm{RP}$ & Total & & & \\
\hline \multicolumn{9}{|l|}{ Tinamidae Gray, 1840} \\
\hline Crypturellus parvirostris (Wagler, 1827) & Inhambu-chororó & & & & & B & B & $\mathrm{B}$ \\
\hline Crypturellus soui (Hermann, 1783) & Tururim & & & & & B & $B$ & $\mathrm{~B}$ \\
\hline Crypturellus tataupa (Temminck, 1815) & Inhambu-chintã & & & & & B & $B$ & M \\
\hline \multicolumn{9}{|l|}{ Cracidae Rafinesque, 1815} \\
\hline Ortalis guttata (Spix, 1825) & Aracuã & & & & & B & B & B \\
\hline \multicolumn{9}{|l|}{ Ardeidae Leach, 1820} \\
\hline Butorides striata (Linnaeus, 1758) & Socozinho & & & & & B & B & B \\
\hline Tigrisoma lineatum (Boddaert, 1783) & Socó-boi & & & & & $\mathrm{M}$ & $B$ & $\mathrm{~B}$ \\
\hline \multicolumn{9}{|l|}{ Cathartidae Lafresnaye, 1839} \\
\hline Cathartes aura (Linnaeus, 1758) & Urubu-de-cabeça-vermelha & & & & & B & B & B \\
\hline Cathartes burrovianus Cassin, 1845 & Urubu-de-cabeça-amarela & & & & & $\mathrm{M}$ & B & M \\
\hline Coragyps atratus (Bechstein, 1793) & Urubu-de-cabeça-preta & & & & & B & $B$ & $\mathrm{~B}$ \\
\hline \multicolumn{9}{|l|}{ Accipitridae Vigors, 1824} \\
\hline Buteo brachyurus Vieillot, 1816 & Gavião-de-cauda-curta & & & & & $\mathrm{M}$ & B & B \\
\hline Buteo nitidus (Latham, 1790) & Gavião-pedrês & & & & & $\mathrm{M}$ & B & B \\
\hline Elanus leucurus (Vieillot, 1818) & Gavião-peneira & & & & & B & B & M \\
\hline Gampsonyx swainsonii Vigors, 1825 & Gaviãozinho & & & & & B & $B$ & $\mathrm{~B}$ \\
\hline Rupornis magnirostris (Gmelin, 1788) & Gavião-carijó & & & & & B & B & B \\
\hline Spizaetus tyrannus (Wied, 1820) & Gavião-pega-macaco & & & & & M & B & $\mathrm{B}$ \\
\hline \multicolumn{9}{|l|}{ Falconidae Leach, 1820} \\
\hline Micrastur gilvicollis (Vieillot, 1817) & Falcão-mateiro & & & & & $A$ & B & B \\
\hline Milvago chimachima (Vieillot, 1816) & Carrapateiro & & & & & B & $B$ & $\mathrm{~B}$ \\
\hline Caracara plancus (Miller, 1777) & Caracará & & & & & B & B & B \\
\hline \multicolumn{9}{|l|}{ Rallidae Rafinesque, 1815} \\
\hline Aramides cajanea (Statius Muller, 1776) & Saracura-três-potes & & & & & A & B & B \\
\hline Laterallus viridis (Statius Muller, 1776) & Sanã-castanha & & & & & B & B & B \\
\hline \multicolumn{9}{|l|}{ Columbidae Leach, 1820} \\
\hline Columbina minuta (Linnaeus, 1766) & Rolinha-de-asa-canela & & & & & B & B & $\mathrm{B}$ \\
\hline Columbina passerina (Linnaeus, 1758) & Rolinha-cinzenta & & & & & B & B & B \\
\hline Columbina talpacoti (Temminck, 1811) & Rolinha-roxa & 2 & & & 2 & B & B & B \\
\hline Leptotila rufaxilla (Richard \& Bernard, 1792) & Juriti-gemedeira & & & & & $\mathrm{M}$ & B & B \\
\hline \multicolumn{9}{|l|}{ Psittacidae Rafinesque, 1815} \\
\hline Forpus xanthopterygius (Spix, 1824) & Tuim & & & & & B & B & B \\
\hline Touit surdus (Kuhl, 1820) & Apuim-de-cauda-amarela & 3 & & & 3 & A & $A$ & A \\
\hline \multicolumn{9}{|l|}{ Cuculidae Leach, 1820} \\
\hline Crotophaga ani Linnaeus, 1758 & Anu-preto & & & & & B & $B$ & B \\
\hline Guira guira (Gmelin, 1788) & Anu-branco & & & & & B & $B$ & $\mathrm{~B}$ \\
\hline Piaya cayana (Linnaeus, 1766) & Alma-de-gato & & & & & B & B & B \\
\hline Tapera naevia (Linnaeus, 1766) & Saci & & & & & B & B & $\mathrm{B}$ \\
\hline & & & & & & & & tinua \\
\hline
\end{tabular}


Tabela I. Continuação.

\begin{tabular}{llllllll}
\multirow{2}{*}{ Espécies } & \multirow{3}{*}{ Nome popular } & \multicolumn{3}{c}{ 2003-2004 } & & \multirow{2}{*}{ PD } & PP \\
\cline { 3 - 4 } & CP & RC & RP & Total & & \\
\hline
\end{tabular}

Strigidae Leach, 1820

Megascops choliba (Vieillot, 1817)

Pulsatrix perspicillata (Latham, 1790)

Nyctibiidae Chenu \& Des Murs, 1851

Nyctibius griseus (Gmelin, 1789)

Caprimulgidae Vigors, 1825

Nyctidromus albicollis (Gmelin, 1789)

Caprimulgus rufus Boddaert, 1783

Caprimulgus parvulus Gould, 1837

Hydropsalis torquata (Gmelin, 1789)

Apodidae Olphe-Galliard, 1887

Tachornis squamata (Cassin, 1853)

Trochilidae Vigors, 1825

Amazilia fimbriata (Gmelin, 1788)

Amazilia versicolor (Vieillot, 1818)

Chlorestes notata (Reich, 1793)

Chlorostilbon lucidus (Shaw, 1812)

Chrysolampis mosquitus (Linnaeus, 1758)

Eupetomena macroura (Gmelin, 1788)

Glaucis hirsutus (Gmelin, 1788)

Phaethornis pretrei (Lesson \& Delattre, 1839)

Phaethornis ruber (Linnaeus, 1758)

Trogonidae Lesson, 1828

Trogon curucui Linnaeus, 1766

Galbulidae Vigors, 1825

Galbula ruficauda Cuvier, 1816

Bucconidae Horsfield, 1821

Nystalus maculatus (Gmelin, 1788)

Picidae Leach, 1820

Colaptes melanochloros (Gmelin, 1788)

Veniliornis passerinus (Linnaeus, 1766)

Picumnus cirratus Temminck, 1825

Picumnus exilis (Lichtenstein, 1823)

Thamnophilidae Swainson, 1824

Dysithamnus mentalis (Temminck, 1823)

Formicivora grisea (Boddaert, 1783)

Herpsilochmus atricapillus Pelzeln, 1868

Herpsilochmus rufimarginatus (Temminck, 1822)

Myrmotherula axillaris (Vieillot, 1817)

Taraba major (Vieillot, 1816)

Thamnophilus caerulescens Vieillot, 1816

Thamnophilus palliatus (Lichtenstein, 1823)

Conopophagidae Sclater \& Salvin, 1873

Conopophaga lineata (Wied, 1831)
Corujinha-do-mato

Murucututu

Mãe-da-lua

Bacurau

João-corta-pau

Bacurau-chintã

Bacurau-tesoura

Tesourinha

Beija-flor-de-garganta-verde

Beija-flor-de-banda-branca

Beija-flor-de-garganta-azul

Besourinho-de-bico-vermelho

Beija-flor-vermelho

Beija-flor-tesoura

Balança-rabo-de-bico-torto

Rabo-branco-acanelado

Rabo-branco-rubro

Surucuá-de-barriga-vermelha

Ariramba-de-cauda-ruiva

Rapazinho-dos-velhos

Pica-pau-verde-barrado

Picapauzinho-anão

Pica-pau-anão-barrado

Pica-pau-anão-de-pintas-amarelas

Choquinha-lisa

Papa-formiga-pardo

Chorozinho-de-chapéu-preto

Chorozinho-de-asa-vermelha

Choquinha-de-flanco-branco

Choró-boi

Choca-da-mata

Choca-listrada

Chupa-dente

B B B

$M \quad B \quad B$

B $\quad$ B $\quad$ B

1

B $B \quad B$

B $\quad$ B $\quad$ B

B B B

B B B

B B $B$

M B B

B $\quad$ B $\quad$ B

B $\quad$ B $\quad$ B

B $B \quad$ B

B $B \quad B$

B $B \quad$ B

B B B

B B B

M B B

$11 \quad M \quad B \quad B$

$\begin{array}{llllll}9 & 8 & 17 & \text { B } & \text { B } & \text { B }\end{array}$

$\begin{array}{lllllllllll}M & B & B\end{array}$

1 1 1 B $\quad$ B $\quad$ B

1 1 1 B $\quad$ B $\quad$ B

B $\quad$ B $\quad M$

$M \quad B \quad B$

$M \quad$ B $B$

$\begin{array}{llllll}8 & 1 & 9 & \mathrm{~B} & \mathrm{~B} & \mathrm{~B}\end{array}$

$51-6-M-B$

$M \quad B \quad B$

$M \quad B \quad B$

B $B$ B

$\begin{array}{lllllll}7 & 1 & 1 & 9 & \mathrm{~B} & \mathrm{~B} & \mathrm{M}\end{array}$

B $B \quad M$

$\begin{array}{llllll}1 & 1 & 2 & \text { M } & \text { A } & \text { M }\end{array}$

Continua

Revista Brasileira de Zoologia 24 (4): 950-964, dezembro 2007 
Tabela I. Continuação.

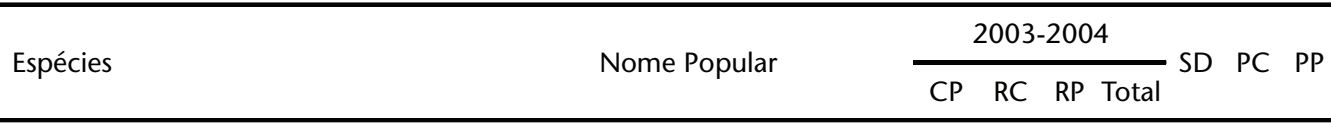

Dendrocolaptidae Gray, 1840

Sittasomus griseicapillus (Vieillot, 1818)

Xiphorhynchus picus (Gmelin, 1788)

Furnariidae Gray, 1840

Certhiaxis cinnamomeus (Gmelin, 1788)

Phacellodomus rufifrons (Wied, 1821)

Synallaxis frontalis Pelzeln, 1859

Synallaxis scutata Sclater, 1859

Xenops minutus (Sparrman, 1788)

Xenops rutilans Temminck, 1821

Tyrannidae Vigors, 1825

Camptostoma obsoletum (Temminck, 1824)

Capsiempis flaveola (Lichtenstein, 1823)

Elaenia cristata Pelzeln, 1868

Elaenia flavogaster (Thunberg, 1822)

Leptopogon amaurocephalus Tschudi, 1846

Mionectes oleagineus (Lichtenstein, 1823)

Myiozetetes similis (Spix, 1825)

Ornithion inerme Hartlaub, 1853

Phyllomyias fasciatus (Thunberg, 1822)

Pitangus sulphuratus (Linnaeus, 1766)

Tolmomyias flaviventris (Wied, 1831)

Elaenia mesoleuca (Deppe, 1830)

Elaenia spectabilis Pelzeln, 1868

Fluvicola nengeta (Linnaeus, 1766)

Hemitriccus margaritaceiventer (d'Orbigny \& Lafresnaye, 1837) Sebinho-de-olho-de-ouro

Hemitriccus zosterops (Pelzeln, 1868)

Lathrotriccus euleri (Cabanis, 1868)

Legatus leucophaius (Vieillot, 1818)

Machetornis rixosa (Vieillot, 1819)

Megarynchus pitangua (Linnaeus, 1766)

Myiarchus ferox (Gmelin, 1789)

Myiarchus swainsoni Cabanis \& Heine, 1859

Myiarchus tuberculifer (d'Orbigny \& Lafresnaye, 1837)

Myiarchus tyrannulus (Statius Muller, 1776)

Myiodynastes maculatus (Statius Muller, 1776)

Myiopagis gaimardii (d'Orbigny, 1839)

Myiopagis viridicata (Vieillot, 1817)

Myiophobus fasciatus (Statius Muller, 1776)

Phaeomyias murina (Spix, 1825)

Poecilotriccus fumifrons (Hartlaub, 1853)

Todirostrum cinereum (Linnaeus, 1766)

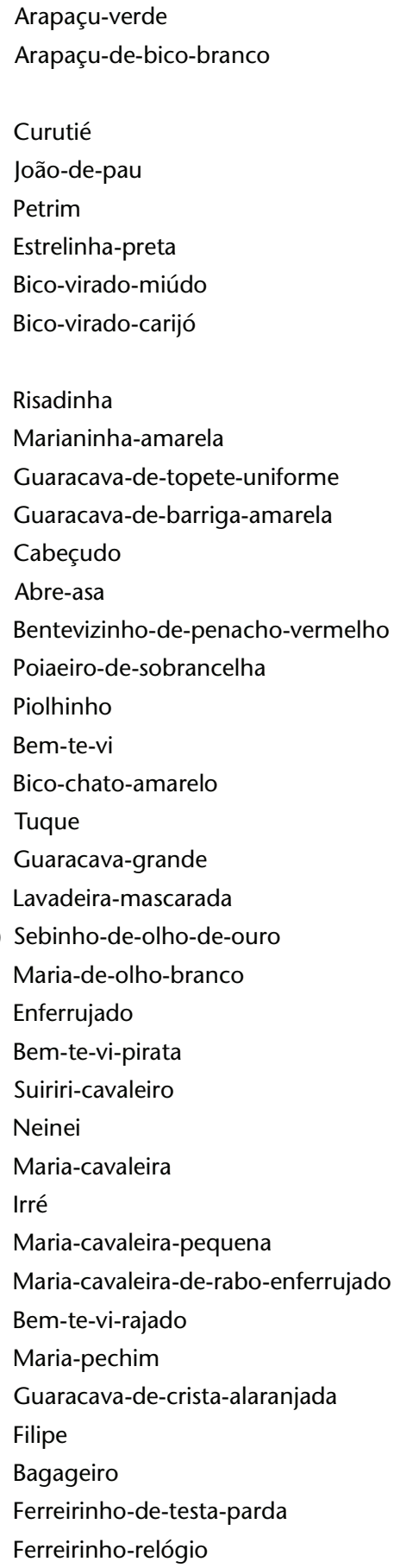

$\begin{array}{ccccccc}6 & 4 & & 10 & \text { M } & \text { B } & \text { M } \\ 5 & 1 & 1 & 7 & \text { B } & \text { B } & \text { B } \\ & & & & & & \\ & & & & \text { M } & \text { B } & \text { B } \\ & & & & \text { M } & \text { B } & \text { B } \\ & & & & \text { B } & \text { B } & \text { B } \\ & & & & \text { M } & \text { M } & \text { M } \\ 7 & 3 & & 10 & \text { M } & \text { B } & \text { B } \\ & & & & \text { M } & \text { B } & \text { B }\end{array}$

2

2

1

11

$9 \quad 14$

5

3

2


Tabela I. Continuação.

\begin{tabular}{|c|c|c|c|c|c|c|c|c|}
\hline \multirow{2}{*}{ Espécies } & \multirow{2}{*}{ Nome Popular } & \multicolumn{4}{|c|}{ 2003-2004 } & \multirow{2}{*}{ SD } & \multirow{2}{*}{ PC } & \multirow{2}{*}{ PP } \\
\hline & & $\mathrm{CP}$ & $\mathrm{RC}$ & $\mathrm{RP}$ & Total & & & \\
\hline Tolmomyias poliocephalus (Taczanowski, 1884) & Bico-chato-de-cabeça-cinza & & & & & M & B & B \\
\hline Tyrannus melancholicus Vieillot, 1819 & Suiriri & & & & & B & B & B \\
\hline Zimmerius gracilipes (Sclater \& Salvin, 1868) & Poiaeiro-de-pata-fina & & & & & M & B & B \\
\hline \multicolumn{9}{|l|}{ Cotingidae Bonaparte, 1849} \\
\hline lodopleura pipra (Lesson, 1831)* & Anambezinho & & & & & M & A & A \\
\hline \multicolumn{9}{|l|}{ Pipridae Rafinesque, 1815} \\
\hline Chiroxiphia pareola (Linnaeus, 1766) & Tangará-falso & 16 & 1 & & 17 & A & B & B \\
\hline Manacus manacus (Linnaeus, 1766) & Rendeira & 26 & 4 & 6 & 36 & B & B & B \\
\hline Neopelma pallescens (Lafresnaye, 1853) & Fruxu-do-cerradão & 28 & 17 & 3 & 48 & M & B & B \\
\hline Pipra rubrocapilla Temminck, 1821 & Cabeça-encarnada & 17 & 1 & & 18 & A & B & B \\
\hline \multicolumn{9}{|l|}{ Tityridae Gray, 1840} \\
\hline Pachyramphus polychopterus (Vieillot, 1818) & Caneleiro-preto & 1 & & & 1 & B & B & B \\
\hline Pachyramphus viridis (Vieillot, 1816) & Caneleiro-verde & 2 & & & 2 & M & B & M \\
\hline \multicolumn{9}{|l|}{ Vireonidae Swainson, 1837} \\
\hline Cyclarhis gujanensis (Gmelin, 1789) & Pitiguari & & & 1 & 1 & B & B & B \\
\hline Vireo olivaceus (Linnaeus, 1766) & Juruviara & 3 & & & 3 & B & B & M \\
\hline \multicolumn{9}{|l|}{ Hirundinidae Rafinesque, 1815} \\
\hline Progne chalybea (Gmelin, 1789) & Andorinha-doméstica-grande & & & & & B & B & B \\
\hline Stelgidopteryx ruficollis (Vieillot, 1817) & Andorinha-serradora & 4 & & & 4 & B & B & B \\
\hline \multicolumn{9}{|l|}{ Troglodytidae Swainson, 1831} \\
\hline Thryothorus genibarbis Swainson, 1838 & Garrinchão-pai-avô & & & & & B & B & B \\
\hline Troglodytes musculus Naumann, 1823 & Corruíra, Rouxinol & 3 & & & 3 & B & B & M \\
\hline \multicolumn{9}{|l|}{ Polioptilidae Baird, 1858} \\
\hline Polioptila plumbea (Gmelin, 1788) & Balança-rabo-de-chapéu-preto & & & & & B & B & B \\
\hline Ramphocaenus melanurus Vieillot, 1819 & Bico-assovelado & 2 & & & 2 & B & B & B \\
\hline \multicolumn{9}{|l|}{ Turdidae Rafinesque, 1815} \\
\hline Turdus amaurochalinus Cabanis, 1850 & Sabiá-poca & & & & & B & B & B \\
\hline Turdus leucomelas Vieillot, 1818 & Sabiá-barranco & 24 & 4 & & 28 & B & B & B \\
\hline Turdus rufiventris Vieillot, 1818 & Sabiá-laranjeira & & & & & B & B & B \\
\hline \multicolumn{9}{|l|}{ Coerebidae d'Orbigny \& Lafresnaye, 1838} \\
\hline Coereba flaveola (Linnaeus, 1758) & Cambacica & 10 & 1 & & 11 & B & B & B \\
\hline \multicolumn{9}{|l|}{ Thraupidae Cabanis, 1847} \\
\hline Cyanerpes cyaneus (Linnaeus, 1766) & Saíra-beija-flor & 15 & 1 & 1 & 17 & B & B & B \\
\hline Dacnis cayana (Linnaeus, 1766) & Saí-azul & 3 & 1 & & 4 & B & B & B \\
\hline Ramphocelus bresilius (Linnaeus, 1766)* & Tiê-sangue & 1 & 1 & 1 & 3 & B & B & M \\
\hline Schistochlamys melanopis (Latham, 1790) & Sanhaçu-de-coleira & & & & & B & B & B \\
\hline Tachyphonus cristatus (Linnaeus, 1766) & Tiê-galo & 1 & & & 1 & M & B & B \\
\hline Tachyphonus rufus (Boddaert, 1783) & Pipira-preta & 10 & 3 & & 13 & B & B & B \\
\hline Tangara cayana (Linnaeus, 1766) & Saíra-amarela & 5 & 1 & & 6 & M & B & B \\
\hline Tersina viridis (Illiger, 1811) & Saí-andorinha & & & & & B & B & M \\
\hline Thlypopsis sordida (d'Orbigny \& Lafresnaye, 1837) & Saí-canário & & & 1 & 1 & B & B & B \\
\hline Thraupis palmarum (Wied, 1823) & Sanhaçu-do-coqueiro & 7 & & & 7 & B & B & B \\
\hline Thraupis sayaca (Linnaeus, 1766) & Sanhaçu-cinzento & 2 & & & 2 & B & B & B \\
\hline
\end{tabular}




\begin{tabular}{|c|c|c|c|c|c|c|c|c|}
\hline \multirow{2}{*}{ Espécies } & \multirow{2}{*}{ Nome Popular } & \multicolumn{4}{|c|}{$2003-2004$} & \multirow{2}{*}{ SD } & \multirow{2}{*}{ PC } & \multirow{2}{*}{ PP } \\
\hline & & $\mathrm{CP}$ & $\mathrm{RC}$ & $\mathrm{RP}$ & Total & & & \\
\hline Emberizidae Vigors, 1825 & & & & & 0 & & & \\
\hline Arremon taciturnus (Hermann, 1783) & Tico-tico-de-bico-preto & 16 & 10 & 6 & 32 & $\mathrm{M}$ & B & B \\
\hline Basileuterus flaveolus (Baird, 1865) & Canário-do-mato & 18 & 12 & & 30 & M & B & B \\
\hline Sicalis flaveola (Linnaeus, 1766) & Canário-da-terra-verdadeiro & & & & & B & B & B \\
\hline Sporophila angolensis (Linnaeus, 1766) & Curió & & & & & B & B & M \\
\hline Sporophila bouvreuil (Statius Muller, 1776) & Caboclinho & & & & & $\mathrm{M}$ & $\mathrm{M}$ & M \\
\hline Sporophila leucoptera (Vieillot, 1817) & Chorão & & & & & B & B & M \\
\hline Sporophila nigricollis (Vieillot, 1823) & Baiano & 1 & & & 1 & B & B & B \\
\hline Volatinia jacarina (Linnaeus, 1766) & Tiziu & & & & & B & B & B \\
\hline \multicolumn{9}{|l|}{ Cardinalidae Ridgway, 1901} \\
\hline Cyanocompsa brissonii (Lichtenstein, 1823) & Azulão & & & & & $\mathrm{M}$ & B & B \\
\hline Saltator maximus (Statius Muller, 1776) & Tempera-viola & 2 & & & 2 & B & B & B \\
\hline \multicolumn{9}{|l|}{ Icteridae Vigors, 1825} \\
\hline Icterus cayanensis (Linnaeus, 1766) & Encontro & 1 & & & 1 & B & B & B \\
\hline Molothrus bonariensis (Gmelin, 1789) & Vira-bosta & 1 & & & 1 & B & B & M \\
\hline \multicolumn{9}{|l|}{ Fringillidae Leach, 1820} \\
\hline Euphonia chlorotica (Linnaeus, 1766) & Fim-fim & & & & & B & B & B \\
\hline Euphonia violacea (Linnaeus, 1758) & Gaturamo-verdadeiro & 11 & 2 & 2 & 15 & B & B & B \\
\hline \multicolumn{9}{|l|}{ Estrildidae Bonaparte, 1850} \\
\hline Estrilda astrild (Linnaeus, 1758) & Bico-de-lacre & & & & & * & * & * \\
\hline \multicolumn{9}{|l|}{ Passeridae Rafinesque, 1815} \\
\hline Passer domesticus (Linnaeus, 1758) & Pardal & & & & & $\mathrm{B}$ & $\mathrm{B}$ & M \\
\hline Total & & 335 & 98 & 23 & 456 & & & \\
\hline
\end{tabular}

(Lafresnaye, 1853) e Turdus leucomelas Vieillot 1818 (todos com $83,33 \%$ ) foram as espécies mais freqüentes. As três famílias mais representativas foram: Pipridae $(26,1 \%)$, Emberizidae $(15,1 \%)$ e Tyrannidae (13,8\%), somando 55\% das aves capturadas.

A menor massa corporal foi de Capsiemps flaveola (Lichtenstein, 1823) (6 g), contrastando com T. leucomelas (75 g). A média de massa corporal foi maior entre machos para $A$. taciturnus $(\mathrm{p}<0,05)$ e o contrário ocorreu com Cyanerpes cyaneus (Linnaeus, 1766) e M. manacus. Não houve diferença significativa com relação aos valores maiores na estação chuvosa para M. manacus e Pipra rubrocapilla Temminck, 1821 e menores para A. taciturnus ( $\mathrm{p}>0,05)$ (Tab. II).

Exceto no diâmetro do tarso em C. cyaneus e tamanho da asa em M. manacus, as medidas médias foram maiores entre machos do que entre fêmeas, com algumas diferenças significativas $(\mathrm{p}<0,05)$ (Tab. III).

Abril e maio de 2004 foram os meses com maior número de mudas de rêmiges. Para retrizes houve mais mudas entre dezembro de 2003 e janeiro de 2004 . A sobreposição de mudas de rêmiges e retrizes demonstrou, de maneira geral, um padrão semelhante dessas mudas ao longo do ano. As de contorno apresentaram padrões irregulares, com picos em outubro e dezembro de 2003 e abril e maio de 2004.

Independente da estação do ano, o maior percentual de muda foi de contorno, seguida de rêmiges e retrizes para os adultos e/ou aqueles com idade indeterminada, quando com plumagem homogênea. Houve associação significativa entre estação de ano/ocorrência de muda $(\mathrm{p}<0,05)$. Os percentuais de mudas de retrizes foram próximos entre a estação chuvosa ou seca e não houve associação significativa entre as duas variáveis ( $\mathrm{p}>0,05)$ (Tab. IV).

Registraram-se placas de incubação em 110 indivíduos, totalizando $24,1 \%$ das amostras, principalmente entre fevereiro e abril de 2004 (a maior parte no período da chuva), com um pico em agosto de 2003, estando mais acentuadas nos meses de novembro de 2003, fevereiro, março e abril de 2004. Em todos os meses houve indivíduos sem placa de choco, com destaque para setembro (Fig. 4). O teste Qui-quadrado apontou diferença significativa entre os meses em relação à ocorrência de placa de incubação $(\mathrm{p}<0,001)$. 
Tabela II. Guildas alimentares, freqüência de ocorrência, variação, médias e desvio padrão das massas corpóreas registradas para as espécies de aves capturadas no RECD entre julho de 2003 e junho de 2004. (GL) Guildas alimentares, (FO) freqüência de ocorrência, (MC) massa corporal, (dp) desvio padrão, (n) número de indivíduos analisados quanto ao peso.

\begin{tabular}{|c|c|c|c|c|c|c|}
\hline \multirow{2}{*}{ Família } & \multirow{2}{*}{ Espécies } & \multirow{2}{*}{$\mathrm{GL}$} & \multicolumn{2}{|c|}{ FO } & \multirow{2}{*}{ Variação MC (g) } & \multirow{2}{*}{ Média $\pm d p(n)$} \\
\hline & & & $\mathrm{n}$ & $\%$ & & \\
\hline Columbidae & Columbina talpacoti & $\mathrm{G}, \mathrm{F}$ & 2 & 16,67 & $48,0-54,0$ & $51,0 \pm 4,2(2)$ \\
\hline Psittacidae & Touit surdus & $\mathrm{F}$ & 2 & 16,67 & $54,0-68,0$ & $62,5 \pm 7,5(3)$ \\
\hline Caprimulgidae & Nyctidromus albicollis & I & 1 & 8,33 & - & - \\
\hline Trogonidae & Trogon curucui & $\mathrm{I}, \mathrm{F}$ & 1 & 8,33 & - & - \\
\hline Galbulidae & Galbula ruficauda & 1 & 5 & 41,67 & $20,5-26,0$ & $23,3 \pm 1,5(16)$ \\
\hline \multirow[t]{2}{*}{ Picidae } & Colaptes melanochloros & $\mathrm{I}, \mathrm{F}$ & 1 & 8,33 & - & - \\
\hline & Veniliornis passerinus & I & 1 & 8,33 & - & - \\
\hline \multirow[t]{3}{*}{ Thamnophilidae } & Formicivora grisea & I & 5 & 41,67 & $10,0-11,5$ & $10,7 \pm 0,6(9)$ \\
\hline & Herpsilochmus atricapillus & 1 & 4 & 33,33 & $8,0-15,0$ & $10,3 \pm 2,5(6)$ \\
\hline & Thamnophilus caerulescens & I & 5 & 41,67 & $10,5-22,0$ & $17,2 \pm 5,0(9)$ \\
\hline Conopophagidae & Conopophaga lineata & I & 1 & 8,33 & $21,0-21,5$ & $21,3 \pm 0,4(2)$ \\
\hline \multirow[t]{2}{*}{ Dendrocolaptidae } & Sittasomus griseicapillus & 1 & 4 & 33,33 & $9,0-14,0$ & $11,6 \pm 1,3(10)$ \\
\hline & Xiphorhynchus picus & I & 6 & 50,00 & $31,0-40,0$ & $35,8 \pm 3,1(7)$ \\
\hline Furnariidae & Xenops minutus & I & 5 & 41,67 & $9,5-17,0$ & $11,2 \pm 2,4(10)$ \\
\hline \multirow[t]{10}{*}{ Tyrannidae } & Camptostoma obsoletum & $\mathrm{I}, \mathrm{F}$ & 1 & 8,33 & $9,0-10,0$ & $9,5 \pm 0,7(2)$ \\
\hline & Capsiempis flaveola & I & 2 & 16,67 & $6,0-9,0$ & $7,5 \pm 2,1(2)$ \\
\hline & Elaenia cristata & $\mathrm{F}, \mathrm{I}$ & 1 & 8,33 & - & - \\
\hline & Elaenia flavogaster & I & 7 & 58,33 & $13,0-24,0$ & $20,1 \pm 3,1(12)$ \\
\hline & Leptopogon amaurocephalus & 1 & 6 & 50,00 & $9,5-14,0$ & $11,7 \pm 1,0(22)$ \\
\hline & Mionectes oleagineus & I & 3 & 25,00 & $11,0-14,0$ & $12,1 \pm 1,3(5)$ \\
\hline & Myiozetetes similis & $\mathrm{F}, \mathrm{I}$ & 3 & 25,00 & $26,0-51,0$ & $42,7 \pm 14,4(3)$ \\
\hline & Ornithion inerme & $\mathrm{F}, \mathrm{I}$ & 2 & 16,67 & $7,0-8,0$ & $7,5 \pm 0,7(2)$ \\
\hline & Pitangus sulphuratus & $\mathrm{O}$ & 1 & 8,33 & - & - \\
\hline & Tolmomyias flaviventris & $\mathrm{I}, \mathrm{F}$ & 7 & 58,33 & $10,0-13,0$ & $12,0 \pm 0,8(16)$ \\
\hline \multirow[t]{4}{*}{ Pipridae } & Chiroxiphia pareola & $\mathrm{F}$ & 7 & 58,33 & $17,5-23,5$ & $19,5 \pm 1,5(17)$ \\
\hline & Manacus manacus & $\mathrm{F}, \mathrm{I}$ & 11 & 91,67 & $12,5-24,0$ & $15,0 \pm 2,2(36)$ \\
\hline & Neopelma pallescens & $\mathrm{I}, \mathrm{F}$ & 10 & 83,33 & $13,0-21,0$ & $18,0 \pm 1,6(48)$ \\
\hline & Pipra rubrocapilla & $\mathrm{F} ?$ & 9 & 75,00 & $11,0-21,5$ & $12,9 \pm 2,4(18)$ \\
\hline \multirow[t]{2}{*}{ Tityridae } & Pachyramphus polychopterus & $\mathrm{I}, \mathrm{F} ?$ & 1 & 8,33 & - & - \\
\hline & Pachyramphus viridis & $\mathrm{I}, \mathrm{F}$ & 2 & 16,67 & $18,0-20,0$ & $19,0 \pm 1,4(2)$ \\
\hline \multirow[t]{2}{*}{ Vireonidae } & Cyclarhis gujanensis & $\mathrm{O}$ & 1 & 8,33 & - & - \\
\hline & Vireo olivaceus & $?$ & 2 & 16,67 & $14,0-16,0$ & $15,0 \pm 1,0(3)$ \\
\hline Hirundinidae & Stelgidopteryx ruficollis & I & 2 & 16,67 & $15,0-16,0$ & $15,6 \pm 0,5(4)$ \\
\hline Troglodytidae & Troglodytes musculus & 1 & 2 & 16,67 & $11,0-12,5$ & $12,0 \pm 0,9(3)$ \\
\hline Polioptilidae & Ramphocaenus melanurus & 1 & 2 & 16,67 & $9,0-10,0$ & $9,5 \pm 0,7(2)$ \\
\hline Turdidae & Turdus leucomelas & $\mathrm{F}, \mathrm{I}$ & 10 & 83,33 & $53,0-75,0$ & $62,3 \pm 5,2(28)$ \\
\hline Coerebidae & Coereba flaveola & $\mathrm{I}, \mathrm{F}, \mathrm{N}$ & 5 & 41,67 & $9,0-16,0$ & $10,2 \pm 2,0(11)$ \\
\hline \multirow[t]{5}{*}{ Thraupidae } & Cyanerpes cyaneus & $\mathrm{N}, \mathrm{I}$ & 5 & 41,67 & $12,0-20,0$ & $13,7 \pm 1,8(17)$ \\
\hline & Dacnis cayana & $\mathrm{F}, \mathrm{I}, \mathrm{N}$ & 2 & 16,67 & $11,0-13,0$ & $12,3 \pm 1,0(4)$ \\
\hline & Ramphocelus bresilius & $\mathrm{F}$ & 2 & 16,67 & $27,5-36,0$ & $30,5 \pm 4,8(3)$ \\
\hline & Tachyphonus cristatus & $\mathrm{F}, \mathrm{I}$ & 1 & 8,33 & - & - \\
\hline & & & & & & Continua \\
\hline
\end{tabular}


Tabela II. Continuação.

\begin{tabular}{|c|c|c|c|c|c|c|}
\hline \multirow{2}{*}{ Família } & \multirow{2}{*}{ Espécies } & \multirow{2}{*}{ GL } & \multicolumn{2}{|c|}{ FO } & \multirow{2}{*}{ Variação MC (g) } & \multirow{2}{*}{ Média $\pm d p(n)$} \\
\hline & & & $n$ & $\%$ & & \\
\hline & Tachyphonus rufus & $\mathrm{F}, \mathrm{l}$ & 6 & 50,00 & $26,0-39,0$ & $31,9 \pm 3,3(13)$ \\
\hline & Tangara cayana & $?$ & 3 & 25,00 & $14,0-22,0$ & $18,2 \pm 2,6(6)$ \\
\hline & Thlypopsis sordida & $\mathrm{F}, \mathrm{I}$ & 1 & 8,33 & - & - \\
\hline & Thraupis palmarum & $\mathrm{I}, \mathrm{F}$ & 3 & 25,00 & $35,5-43,0$ & $38,6 \pm 2,7(7)$ \\
\hline & Thraupis sayaca & $\mathrm{F}$ & 2 & 16,67 & $34,5-35,0$ & $34,8 \pm 0,4(2)$ \\
\hline \multirow[t]{3}{*}{ Emberizidae } & Arremon taciturnus & G & 10 & 83,33 & $24,0-31,0$ & $26,4 \pm 1,5(32)$ \\
\hline & Basileuterus flaveolus & I & 9 & 75,00 & $10,0-14,0$ & $12,5 \pm 0,9(30)$ \\
\hline & Sporophila nigricollis & G & 1 & 8,33 & - & - \\
\hline Cardinalidae & Saltator maximus & G & 2 & 16,67 & $41,0-44,0$ & $42,5 \pm 2,1(2)$ \\
\hline \multirow[t]{2}{*}{ Icteridae } & Icterus cayanensis & $\mathrm{N}, \mathrm{I}$ & 1 & 8,33 & - & - \\
\hline & Molothrus bonariensis & $?$ & 1 & 8,33 & - & - \\
\hline Fringillidae & Euphonia violacea & $\mathrm{F}$ & 5 & 41,67 & $13,0-16,0$ & $14,0 \pm 0,9(15)$ \\
\hline
\end{tabular}
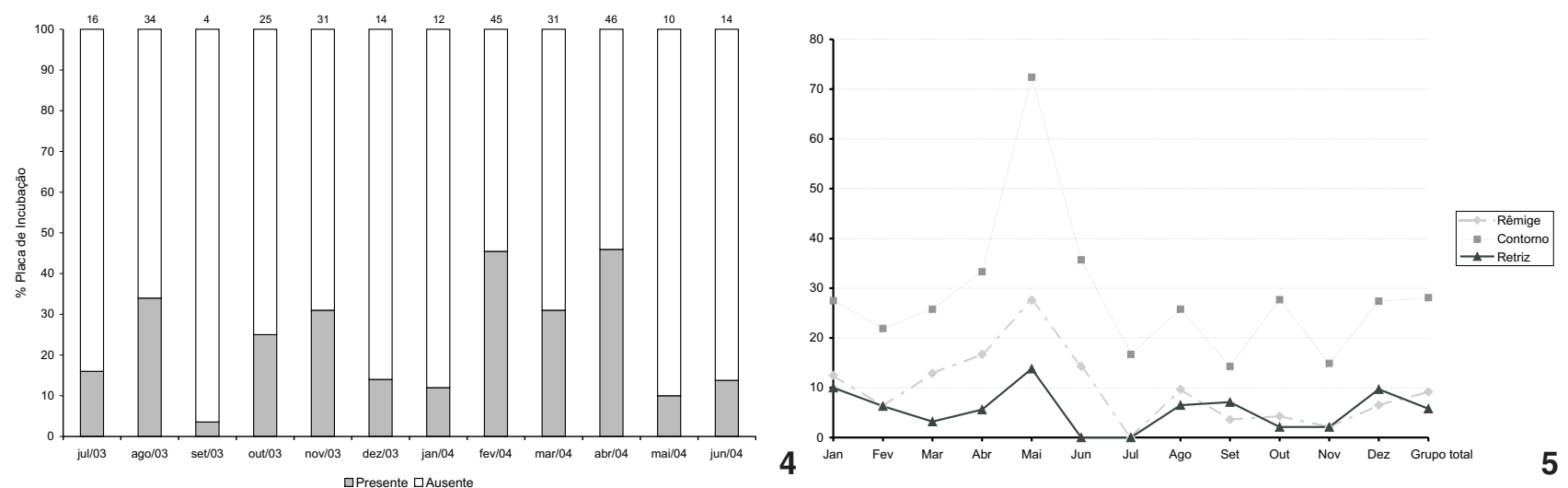

Figuras 4-5. (4) Freqüência percentual da presença e ausência de Placa de Incubação ao longo dos meses de estudo; (5) freqüência percentual de placa e muda simultâneos (sobreposição) por tipo, segundo o mês de ocorrência.

Tabela III. Média e desvio padrão das medidas: asa, tarso e diâmetro do tarso de três espécies de aves segundo o sexo dos animais. (n) Número de indivíduos analisados por espécie e sexo.

\begin{tabular}{|c|c|c|c|c|}
\hline \multirow{2}{*}{ Espécie } & \multirow{2}{*}{ Medida } & Fêmea & Macho & \multirow{2}{*}{ Valor de $\mathrm{p}^{*}$} \\
\hline & & Média \pm Desvio Padrão (n) & Média \pm Desvio Padrão (n) & \\
\hline \multicolumn{5}{|l|}{ Arremon taciturnus } \\
\hline & Asa & $69,55 \pm 2,85(5 / 8)$ & $75,55 \pm 1,88(13 / 24)$ & $<0,0001$ \\
\hline & Tarso & $24,09 \pm 0,60(5 / 8)$ & $24,42 \pm 0,62(13 / 24)$ & 0,3191 \\
\hline & Diâmetro do tarso & $2,30 \pm 0,16(5 / 8)$ & $2,33 \pm 0,15(13 / 24)$ & 0,6799 \\
\hline \multirow[t]{3}{*}{ Cyanerpes cyaneus } & Asa & $62,96 \pm 1,43(6 / 6)$ & $66,26 \pm 1,62(9 / 11)$ & 0,0014 \\
\hline & Tarso & $14,45 \pm 0,29(6 / 6)$ & $14,49 \pm 0,39(9 / 11)$ & 0,8003 \\
\hline & Diâmetro do tarso & $1,89 \pm 0,12(6 / 6)$ & $1,69 \pm 0,14(9 / 11)$ & 0,0127 \\
\hline \multirow[t]{3}{*}{ Manacus manacus } & Asa & $54,50 \pm 3,95(15 / 24)$ & $49,04 \pm 1,10(7 / 10)$ & $<0,0001^{* *}$ \\
\hline & Tarso & $19,37 \pm 0,42(16 / 24)$ & $20,81 \pm 0,55(7 / 10)$ & $<0,0001$ \\
\hline & Diâmetro do tarso & $1,68 \pm 0,11(16 / 24)$ & $1,69 \pm 0,14(7 / 10)$ & 0,9582 \\
\hline
\end{tabular}

* Teste t-Student; ** Teste t-Student com variâncias desiguais. 
Tabela IV. Avaliação da ocorrência de cada tipo de muda segundo a estação da captura.

\begin{tabular}{|c|c|c|c|c|c|c|c|c|}
\hline \multirow{3}{*}{ Tipo de muda } & \multirow{3}{*}{ Estação do ano } & \multicolumn{4}{|c|}{ Muda } & & & \multirow{3}{*}{ Valor de $\mathrm{p}^{\star}$} \\
\hline & & \multicolumn{2}{|c|}{ Sim } & \multicolumn{2}{|c|}{ Não } & \multicolumn{2}{|c|}{ Total } & \\
\hline & & $\mathrm{n}$ & $\%$ & $\mathrm{n}$ & $\%$ & $\mathrm{n}$ & $\%$ & \\
\hline \multirow[t]{2}{*}{ Rêmiges } & Chuvosa & 36 & 24,2 & 113 & 75,8 & 149 & 100,0 & $<0,0001$ \\
\hline & Seca & 21 & 7,2 & 270 & 92,8 & 291 & 100,0 & \\
\hline Grupo total & & 57 & 12,9 & 383 & 87,1 & 440 & 100,0 & \\
\hline \multirow[t]{2}{*}{ Contorno } & Chuvosa & 83 & 55,7 & 66 & 44,3 & 149 & 100,0 & $<0,0001$ \\
\hline & Seca & 92 & 31,6 & 199 & 68,4 & 291 & 100,0 & \\
\hline Grupo total & & 175 & 39,8 & 265 & 60,2 & 440 & 100,0 & \\
\hline \multirow[t]{2}{*}{ Retrizes } & Chuvosa & 12 & 8,1 & 137 & 91,9 & 149 & 100,0 & 0,7555 \\
\hline & Seca & 26 & 8,9 & 265 & 91,1 & 291 & 100,0 & \\
\hline Grupo total & & 38 & 8,6 & 402 & 91,4 & 440 & 100,0 & \\
\hline
\end{tabular}

* Teste Qui-quadrado de Pearson.

Associado à placa, o percentual de muda de contorno (40,0\%), de rêmige $(12,9 \%)$ e retriz $(8,5 \%)$, foi sempre mais elevado. Os percentuais daqueles com muda de rêmige e retriz foi mais elevado em $2,7 \%$ e $3,6 \%$, respectivamente, entre animais com placa, sem associação significativa $(p>0,05)$. Para contorno, o percentual foi $11,4 \%$ mais elevado entre os que tinham placa, com associação significativa ( $<<0,05)$ (Fig. 5).

O percentual de placa e muda simultâneos foi maior $(28,1 \%)$ para muda de contorno e menor $(5,8 \%)$ para retriz. $\mathrm{Na}$ análise mensal os percentuais de placa e muda de contorno foram mais elevados do que os demais. Para rêmiges, os meses em destaque foram: maio $(27,6 \%)$, abril $(16,7 \%)$, junho $(14,3 \%)$ e janeiro de 2004 (12,5\%). Para contorno, o maior percentual foi em maio de 2004 (72,4\%), enquanto os demais meses variaram de $14,3 \%$ até $35,7 \%$. Para retriz os dois maiores percentuais foram em maio (13,8\%) e janeiro de 2004 (10,0\%).

Na análise de Componentes Principais, observou-se que o eixo I esteve positivamente ligado, sobretudo, à Basileuterus flaveolus (Baird, 1865), com maior freqüência em julho de 2003, maio e junho de 2004, relacionado ao período de maiores precipitação e umidade (coordenadas positivas das variáveis precipitação e umidade). Em oposição projetaram-se negativamente ao eixo I as espécies $P$. rubrocapilla, $T$. flaviventris e M. manacus (outubro a dezembro de 2003 e janeiro de 2004), onde M. manacus destacou-se junto a variável temperatura (Fig. $6)$.

Das espécies identificadas na listagem geral do RECD, seis apresentaram alta sensibilidade aos distúrbios ambientais e três com alta prioridade de conservação, sendo duas destas com alta prioridade de pesquisa (PARKER III et al. 1996). Quatro encontravam-se ameaçadas de extinção e três são endêmicas para a região (Tab. I).

Para as espécies capturadas entre 2003 e 2004, as onívoras destacaram-se das demais guildas alimentares $(40,1 \%)$, seguida dos insetívoros (36\%). Os granívoros tiveram o menor

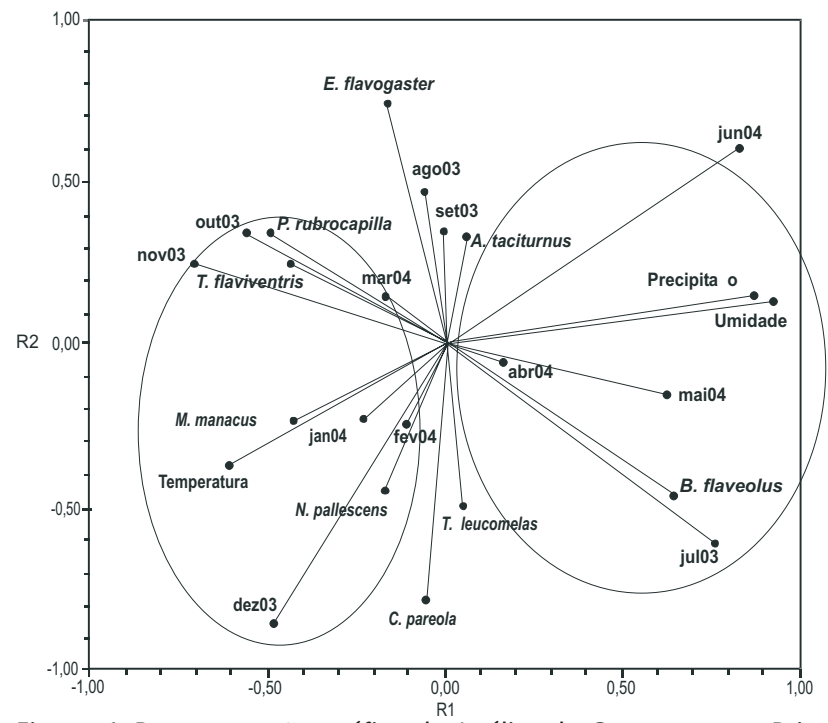

Figura 6. Representação gráfica da Análise de Componentes Principais, utilizando-se o método de ordenação e projeção. Relação entre meses de capturas, espécies mais representativas e fatores abióticos (temperatura e umidade).

registro $(0,7 \%)$ para este método de captura.

Foram capturados três indivíduos de Touit surdus e dois de Ornithion inerme. Os primeiros foram capturados em dois momentos distintos, porém todos na mesma rede, disposta entre dois sapotizeiros (Achras sapota Linnaeus), de altura inferior a quatro metros, onde os mesmos forrageavam. Fato semelhante ocorreu com Ornithion inerme, primeiro registro com captura para o Estado. Ambos apresentavam muda de contorno, mas apenas um substituía sua nona rêmige primária e indicava placa de choco em estágio final de sucessão, com o período de reprodução terminando ou já concluído. 


\section{DISCUSSÃO}

As recuperações demonstraram fidelidade de algumas aves ao ambiente, reforçando a importância dos pequenos fragmentos para espécies residentes e ocasionais e permitiram registrar o tempo de anilha. SNow \& Lill (1974) relataram a idade para M. manacus em mais de 14 anos e Lopes et al. (1980) apresentaram o tempo de anilha para várias espécies. Apenas em duas espécies (M. manacus e Thamnophilus caerulescens) as pesquisas coincidiram e nenhum tempo de anilha apresentado por aqueles autores foi maior do que neste trabalho.

A composição da avifauna do RECD foi semelhante a outros trabalhos em matas pernambucanas, como: LyRA-Neves et al. (2000), 187 espécies para a Área de Proteção Ambiental de Guadalupe, área superior a 60 ha e Lyra-Neves et al. (2004) na Reserva Estadual de Gurjaú, 220 espécies registradas em 1077,10 ha. Essa semelhança pode estar relacionada à presença de florestas vizinhas ao RECD, como a Usina São José (4 km da área estudada). Esta proximidade certamente contribui com a existência de muitas espécies que sobrevivem em pequenas áreas interligadas a plantações, pastagens e outros fragmentos, ampliando o uso de recurso por esses animais (Aleixo \& Galetti 1997, MaldonaDo-Coelho \& MARINI 2000, Telino-JúnIOR et al. 2000).

Baixos valores de FO e muitas espécies com um único registro eram esperados devido ao método amostral e às observações dos padrões fitogeográficos (fragmento em regeneração), que limitou as capturas às espécies que ocupam o estrato baixo da floresta.

Mais de 50\% das espécies capturadas foram das famílias Pipridae, Emberizidae e Tyrannidae. Em geral, espécies mais numerosas são aquelas com vasta distribuição que habitam, sobretudo, a mata e a capoeira. TERBORGH (1992) afirmou ser incerto o futuro de muitas espécies nas "sobras" de habitats fragmentados, que podem prover bem determinadas espécies, sendo insuficientes para outras. M. manacus, N. pallescens e A. taciturnus, espécies frugívoras/insetívoras e granívora, foram mais representativas devido ao efeito de borda. A alta luminosidade neste ambiente propicia maior produção de frutos e de plantas invasoras produtoras de sementes, que são a base alimentar destas espécies (DÁrio et al. 2002). Já espécies especialistas como dendrocolaptídeos e pica-paus (escaladores de troncos) e formicarídeos (seguidores de correição), tendem a diminuir suas populações em fragmentos pequenos (BierregaARD et al. 1992).

Alguns estudos prévios (Clark JúNIOR 1979, ONIKI 1981, Dunning 1987, Oniki \& Willis 1993, Belton 1994, Reinert et al. 1996, Sick 1997) geraram dados sobre massa corpórea de aves do mundo. O presente estudo, ao comprovar diferenças entre pesos de machos e fêmeas, corrobora com a literatura pesquisada. Baldwin \& Kendeigh (1938) e Clark Júnior (1979) registraram essas desigualdades e comprovaram que a relação não está distribuída de maneira constante, fato observado nesta pesquisa em duas das três espécies analisadas que não apresentaram diferenças significativas na variação de peso entre sexos. Nas comparações entre massa corpórea/estações do ano, embora não tenham existido valores significativos, variações interespecíficas e alterações individuais são comuns (BALDWIN \& Kendeigh 1938). Ou seja, mudanças de peso ocorreram tanto dentro da espécie quanto no indivíduo, variando ao longo do dia e do ano e estão relacionadas a outras variáveis, como: diferenças na temperatura do ar, quantidade de alimentação, migração e outras, não sendo possível estabelecer um padrão. A maneira e extensão de flutuação de peso observada evidenciaram que as aves em seu ajustamento fisiológico são altamente sensíveis a influências ambientais e que esta inter-relação entre função e ambiente afeta extremamente esse comportamento (Baldwin \& Kendeigh 1938).

Para mudas, o presente estudo confirma a literatura quanto à simultaneidade entre mudas de rêmige e retriz (Poulin et al. 1992, Sick 1997, Marini \& DuRães 2001). As penas de contorno não seguiram esse padrão, concordando com MARINI \& DURÃES (2001), provavelmente devido ao critério empregado (três penas em crescimento na cabeça, dorso e/ou ventre) para afirmar a existência de muda pós-nupcial, o que pode mascarar a análise. Marini \& Durães (2001) aumentaram este critério, concluindo que este tipo de muda ocorre ao longo do ano, propondo que estas mudas não estejam necessariamente relacionadas com a muda pós-nupcial. A despeito de diferenças metodológicas, fato semelhante ocorreu neste estudo. Sugere-se o emprego apenas das penas primárias para avaliar o ciclo de muda pósnupcial (Miller 1961, Poulin et al. 1992).

No ciclo anual das aves, reprodução e mudas são eventos que demandam grande energia, levando vários autores a apontar a ausência de simultaneidade entre mudas primárias e condições reprodutivas em regiões temperadas (Miller 1961, PAYNE 1969, Poulin et al.1992). Para aves tropicais, a sobreposição parece ser mais comum (Foster 1975). Neste trabalho não houve comprovação estatística da sobreposição das mudas, possivelmente devido ao reduzido número de espécimes capturados. Todavia, houve correlação negativa entre retriz e placa de incubação e rêmige e placa de incubação, o que está de acordo com os achados dos autores citados.

Quanto aos hábitos alimentares SIgRIST (2006) classifica os piprídeos (M. manacus e N. pallescens) e o T. leucomelas como frugívoros/insetívoros, o que os torna bastante generalistas. A estrutura da comunidade de aves é drasticamente alterada em fragmentos menores. Insetívoros especializados (como insetívoros de tronco e seguidores de correição) cuja captura em rede de neblina é significativa para áreas contínuas, reduz dramaticamente (BIERREGAARD et al. 1992), como observado nesta pesquisa. Com o tempo, a maioria das espécies insetívoras presentes nesses fragmentos, que forrageiam em conjunto com outras espécies, desaparece em fragmentos menores de 10 hectares (Powell 1985, Bierregaard \& Lovejoy 1989).

O aumento das capturas no período de maior oferta alimentar para B. flaveolus sugere explicação para a alta relação observada frente aos dados abióticos. Neste período, percebeu- 
se maior quantidade de insetos, possivelmente os que necessitam de água para completar seu ciclo de vida. Poulin et al. (1992) relataram fato similar em relação à abundância de artrópodes na estação chuvosa. As aves seguem este padrão, com mudas e período reprodutivo nas estações úmidas, quando há oferta de alimentos. Isto pode explicar a relação existente entre esta espécie e os padrões de precipitação e umidade, embora Lefebvre et al. (1992) afirmem que, para regiões tropicais, a disponibilidade de insetos é relativamente constante ao longo do ano. Já M. manacus apresentou maior relação com a temperatura. Isso pode ser explicado quando se consideram períodos mais quentes que antecedem a época reprodutiva. Estas aves utilizam poleiros de exibição para atrair as fêmeas em período pré-nupcial (SICK 1997, SILVA \& RÊGo 2004), sendo mais comum a sua visualização e deslocamento, aumentando a chance de captura. Este fator também pode estar relacionado com a frutificação que ocorre no período após as chuvas, a exemplo das melastomatáceas, muito utilizadas por pequenos frugívoros, como os Pipridae (Galetti \& STотz 1996).

São escassas as informações acerca do Touit surdus e do Ornithion inerme em Pernambuco. O único registro de pele data de dezembro de 1944, quando Berla $(1946,1954)$ coletou três exemplares da espécie nos arredores do Recife. Entre 1996 e 1997, estudos no RECD acrescentaram informações sobre $T$. surdus por meio de observações (TELINo Júnior et al. 2000), com relato de até 40 indivíduos sobrevoando a área, identificados pela vocalização. No presente estudo, perto de 12 indivíduos foram avistados se deslocando. Apesar de conviverem em bando nas copas das árvores (Telino JúnIor et al. 2000), podem ter hábitos de forrageamento mais restrito a pequenos grupos, atraídos por frutos, chegando a estratos inferiores da floresta, como descrito por Sick (1997) para a família dos Psittacidae, inclusive atingindo o solo atrás de cocos ou sementes caídos.

Em 2002, José Fernando Pacheco e Ricardo Parrini observaram um único exemplar de O. inerme em Pernambuco, identificado por vocalização na Reserva Ecológica de Dois Irmãos, Recife, ampliando a listagem de aves para o Parque (Azevedo JúNIOR et al. 1998, FARIAS et al. 2002). Até então, era assinalado apenas para regiões fronteiriças com Alagoas e Paraíba (FARIAS et al. 2002).

O emprego de captura em rede apresenta algumas respostas, sendo suficiente para pesquisas pontuais. Porém com o intuito de maximizar essas respostas, sugere-se seu emprego concomitante com observações acústicas e visuais. A ampliação do montante de dados referentes à composição da avifauna e sua manutenção em fragmentos florestais contribuem para o planejamento de estratégias de conservação dessas áreas que são, muitas vezes, os últimos redutos para a fauna.

\section{CONSIDERAÇÕES FINAIS}

Embora as capturas tenham sido por amostragem, a presença de espécies ameaçadas de extinção com registro em apenas uma área em Pernambuco e a presença de psitacídeos e trogonídeos, que têm dietas especializadas e maior necessidade de áreas extensas com suprimento de frutos disponível todo o ano, comprovam a importância do fragmento na conservação da diversidade biológica da região.

A sobreposição entre mudas de rêmige e retriz sugere que devido ao período reprodutivo prolongado nos trópicos e a presença das placas de incubação no final da estação seca e início da estação chuvosa, haja uma possível sobreposição destas atividades.

Pesquisas em ambientes alterados, principalmente os de tamanho reduzido, são de extrema importância para comprovar a sustentabilidade das espécies, gerando a necessidade de maior coordenação na conservação e manejo racional pelo desenvolvimento de políticas públicas que visem estabelecer planos de manejo que gerenciem essas áreas e regiões afins, evitando que tamanha degradação cause transformações irreversíveis para a estrutura das comunidades em geral.

\section{AGRADECIMENTOS}

Ao Programa de Pós-graduação em Biologia Animal (CCB/ UFPE) e a CAPES pela bolsa concedida à Vivyanne Santiago Magalhães. A Maria L. Santos e Edmilson Mazza, professores de estatística da UFPE, pela valiosa ajuda nas análises e a todos que contribuíram para a realização deste trabalho.

\section{REFERÊNCIAS BIBLIOGRÁFICAS}

Aleixo, A. \& M. GaletTr. 1997. The conservation of the avifauna in a lowland Atlantic forest in south-east Brazil. Bird Conservation International 7: 235-261.

Anciães, M. \& M.Â. Marini. 2000a. Assimetria flutuante em passeriformes da mata Atlântica, p. 187-204. In: M.A. Dos S. Alves; J.M.C. Da Silva; M. van Sluys; H. De G. Bergallo \& C.F.D. DA Rocha (Eds). A ornitologia no Brasil: pesquisa atual e perspectivas. Rio de Janeiro, EDUERJ, 352p.

AnCIÃES, M. \& M.Â. Marini. 2000b. The effects of fragmentation on fluctuating asymmetry in passerine birds of Brazilian tropical forests. Journal of Applied Ecology 37: 1013-1028.

Andrade-Lima, D. DE. 1960. Estudos fitogeográficos de Pernambuco. Arquivos do Instituto de Pesquisas Agronômicas 5: 305-341.

Avery, M.L. 1985. Annual molt pattern in a Malaysian population of fantail warblers (Cisticola juncidis). The Condor 87: 346349.

Azevedo-Júnior, S.M. de; A.G.M. Coelho; M.E. de Larrazábal; R.M. De Lira Neves \& W.R. Telino Júnior. 1998. Conservação e diversidade das aves da Reserva Ecológica de Dois Irmãos, p. 241-250. In: I.C. Machado, A.V. Lopes \& K. C. Pôrto (Eds). Reserva Ecológica de Dois Irmãos: estudos em um remanescente de Mata Atlântica em área urbana (Recife, Pernambuco, Brasil). Recife, Secretaria de Ciência, Tecnologia e Meio Ambiente, 326p.

Azevedo-Júnior, S.M. DE; M.M. Dias; M.E.L. de larrazábal; W.R.

Revista Brasileira de Zoologia 24 (4): 950-964, dezembro 2007 
Telino-Júnior; R.M. de Lyra-Neves \& C.J.G. Fernandes. 2001. Recapturas e recuperações de aves migratórias no litoral de Pernambuco, Brasil. Ararajuba 9 (1): 23-42.

Baldwin, P. \& C. Kendeigh. 1938. Variations in the weight of birds. Auk 55: 416-467.

Belton, W. 1994. Aves do Rio Grande do Sul: distribuição e biologia. São Leopoldo, Editora UNISINOS, 584p.

Berla, H.F. 1946. Lista das colecionadas em Pernambuco, com descrição de uma subespécie n., de um alótipo e notas de campo. Boletim do Museu Nacional do Rio de Janeiro 65: $1-35$.

Berla, H.F. 1954. Um novo (Psittacidae) do nordeste brasileiro (Aves, Psittaciformes). Revista Brasileira de Biologia 14: 59-60.

Bierregaard Jr, R.O. \& T.E. Lovejoy. 1989. Effects of forest fragmentation on Amazonia understory bird communities. Acta Amazonica 19: 215-241.

BierregaArd Jr, RO; T.E. Lovejoy; V. Kapos; A.A. Dos Santos \& R.W. Hutchings. 1992. The biological dynamics of tropical rainforest fragments: a prospective comparison of fragments and continuous forest. BioScience 42 (11): 859-866.

Brown, K.S. JR \& G.G. Brown. 1992. Habitat alteration and species loss in Brazilian forests, p. 119-142. In: T.C. WhitMore \& J.A. SAYER (Eds). Tropical deforestation and species extinction. London, Chapman and Hall, 156p.

Chiarello, A.G. 1999. Effects of fragmentation of the Atlantic forest on mammal communities in south-eastern Brazil. Biological Conservation 89: 71-82.

Clark Júnior, G.A. 1979. Body weights of birds: a review. The Condor 81: 193-202.

Comitê Brasileiro de Registros Ornitológicos. 2006. Listas das aves do Brasil. Versão 15/7/2006. Disponível em: http://www. cbro.org.br [Acesso em 01/IV/2007].

Dário, F.R.; M.C.V. de Vicenzo \& A.F. de Almeida. 2002. Avifauna em fragmentos da Mata Atlântica. Ciência Rural 32 (6): 989 996.

DunNing, J.S. 1987. South America birds: a photographic aid to identification. Pennsylvania, Harrowood Books, 364p.

Farias, G.B. De; M.T. de Brito \& G.L. Pacheco. 2002. Registros ornitológicos de Pernambuco. Recife, Observadores de Aves de Pernambuco, 67p.

Fundação para o Desenvolvimento da Região Metropolitana do ReCIFE. 1993. Monitoramento das reservas ecológicas da região metropolitana do Recife. Recife, Governo do Estado de Pernambuco, Secretaria de Planejamento do Estado de Pernambuco, Fundação de Desenvolvimento da Região Metropolitana, 55p.

Fonseca, G.A.B. DA. 1985. The vanishing Brazilian Atlantic Forest. Biological Conservation 34: 17-34.

Foster, M.S. 1975. The overlap of molting and breeding in some tropical birds. The Condor 77: 304-314.

Galetti, M. \& D. Stotz. 1996. Miconia hypoleuca (Melastomataceae) como espécie-chave para aves frugívoras no sudoeste do Bra- sil. Revista Brasileira de Zoologia 56 (2): 435-439.

Gonzaga de CAmpos. 1912. Mapa florestal. Rio de Janeiro, Serviço Geológico de Mineralogia do Brasil.

Iвама. 1994. Manual de anilhamento de aves silvestres. Brasília, IBAMA, $2^{\mathrm{a}}$ ed., $146 \mathrm{p}$.

Iвама. 2003. Ecossistemas brasileiros. Disponível em: http:// www.ibama.org.br [Acesso em 21.VIII.2003]

InMET. 2004. Seção de Observação e Meteorologia Aplicada. Recife, Instituto Nacional de Meteorologia, SEOMA, 2p.

LAURANCE, W.F. 1991. Edges effects in tropical forest fragments: application of a model for the design of nature reserves. Biological Conservation 57: 205-219.

Lefebvre, G.; B. Poulin \& R. McNeil. 1992. Settlement period and function of long-term territory in tropical mangrove passerines. The Condor 94: 83-92.

Lima, M.L.F. DA C. 1998. A Reserva da Biosfera da Mata Atlântica em Pernambuco - situação atual, ações e perspectivas. São Paulo, Instituto Florestal, Caderno 12, 43p.

Lopes, O. DE S; L. De A. Sacchetta \& E. Dente. 1980. Longevity of wild birds obtained during a banding program in SP, Brasil. Journal of Field Ornithology 51 (2): 144-148.

Lyra-Neves, R.M. de; A.M.I. de Farias; W.R. Telino-Júnior; M. Arzua; M.C.N. Botelho \& M. DA C. Abreu e Lima. 2000. Ectoparasitismo em aves silvestres (Passeriformes - Emberizidae) de Mata Atlântica, Igarassu, Pernambuco. Melopsittacus 3 (2): 64-71.

Lyra-Neves, R.M. de; M.M. Dias; S.M. de Azevedo-Júnior; W.R. Telino-Júnior \& M.E.L. de Larrazábal. 2004. Comunidade de aves da Reserva de Gurjaú, Pernambuco, Brasil. Revista Brasileira de Zoologia 21 (3): 581-592.

Maldonado-Coelho, M.\& M.Â. Marini. 2000. Effects of forest fragment size and successional stage on mixed-species bird flocks in southeastern Brazil. The Condor 102: 585-594.

Marini, M.Â. \& R. Durães. 2001. Annual patterns of molt and reproductive activity of passerines in south-central Brazil. The Condor 103: 767-775.

McNeIL, R. 1971. Lean-season fat in a South American population of Black-necked stilts. Condor 73: 472-475.

Miller, A.H. 1961. Molt cycles in Equatorial Andean sparrows. The Condor 63: 143-161.

Morellato, L.P.C. \& C.F.B. Haddad. 2000. Introduction: the Brazilian Atlantic forest. Biotropica 32 (4b): 786-792.

Myers, N.; R.A. Mittermeier; C.G. Mittermeier; G.A.B. Fonseca \& J. Kent. 2000. Biodiversity hotspots for conservation priorities. Nature 403: 853-858.

ONIKI, Y. 1981. Weights, cloacal temperatures, plumage and molt condition of birds in the state of São Paulo. Revista Brasileira de Biologia 41 (2): 451-460.

Oniki, Y. \& E.O. Willis. 1993. Pesos, medidas, mudas, temperaturas cloacais e ectoparasitos de aves da Reserva Ecológica do Panga, Minas Gerais, Brasil. Boletim do Centro de Estudos Ornitológicos 9: 2-10.

Parker III, T.A.; D.E. Stotz \& J.W. FitzPatrick. 1996. Ecological and distributional databases, p. 113-436. In: D.F. Sтотz; J.W. 
FitzPatrick; T.A. Parker III \& D.K. Moskovits (Eds). Neotropical birds: ecology and consertation. Chicago, University of Chicago Press, XI+700p.

PAYNE, R.B. 1969. Overlap of breeding and molting schedules in a collection of African birds. The Condor 71: 140-145.

Poulin, B.; G. Lefebvre \& R. McNeil. 1992. Tropical avian phenology in relation to abundance and exploitation of food resources. Ecology 73 (6): 2295-2309.

Powell, G.V.N. 1985. Sociobiology and adaptive significance of interspecific foraging flock in the neotropics, p. 713-732. In: P.A. Buckley, M.S. Foster, E.S. Morton, R.S. Ridgely, and F.G. BuCKLey (Eds). Neotropical Ornithology. Washington, American Ornithologists Union, 1041p.

Ranta, P.; T. Blom; J. Niemelã; E. Joensuu \& M. Sittonen. 1998. The fragmented Atlantic rain forest of Brazil: size, shape and distribution of forest fragments. Biodiversity and Conservation 7: 385-403.

Reinert, B.L.; J.C. Pinto; M.R. Bornschein; M. Pichorim \& M.Â. MARINI. 1996. Body masses and measurements of birds from Southern Atlantic Forest, Brazil. Revista Brasileira de Zoologia 13 (4): 815-824.

Ridgely, R.S. \& G. Tudor. 1994a. The birds of South America: the oscines passerines. Austin, University of Texas Press, $2^{\text {nd }}$ ed., $516 \mathrm{p}$.

Ridgely, R.S. \& G. Tudor. 1994b. The birds of South America: the suboscines passerines. Austin, University of Texas Press, $814 \mathrm{p}$.

SICK, H. 1997. Ornitologia Brasileira. Rio de Janeiro, Editora Nova Fronteira, II+862p.

Sigrist, T. 2006. Aves Brasileiras: uma visão artística. Valinos, Editora Avis Brasilis, 672p.

Silva, J.M.C.\& M. TABarelli. 2000. Tree species impoverishment and the future flora of the Atlantic Forest of Northeast Brazil.
Nature 404: 72-74.

SILvA, W.A. DE G. \& P.S. Do RÊGO. 2004. Conservação do soldadinho-do-araripe Antilophia bokermanni (Aves: Pipridae): subsídios para a elaboração do plano de manejo. Curitiba, Observadores de Aves de Pernambuco, Fundação O Boticário de Proteção à Natureza, 32p.

SNOW, DW \& A LiLl. 1974. Longevity records for some neotropical land birds. The Condor 76: 262-267.

SouZA, D.G.S. 2002. All the birds of Brazil: an identification guide. São Paulo, Editora DALL, 356p.

TABARelli, M. 2000. Dois Irmãos: o desafio da conservação biológica em um fragmento de floresta tropical, p. 311-323, In: I.C. Machado; A.V. Lopes \& K.C. Pôrto (Eds). Reserva Ecológica de Dois Irmãos: Estudos em um Remanescente de Mata Atlântica em Área Urbana (Recife, Pernambuco, Brasil). Recife, Editora Universitária, SECTMA, 326p.

Telino-Júnior, W.R.; R.M. De Lyra-Neves \& R.S. Carneiro. 2000. Observações de Touit surdus (Psittacidae) em fragmentos florestais de Pernambuco, Brasil. Melopsittacus 3 (4): 159-165.

Terborgh, J. 1992. Maintenance of the diversity in tropical forests. Biotropica 24: 283-292.

Terborgh, J.; S.K. Robinson; T.A. Parker III; C.A. Munn \& N. Pierpont. 1990. Structure and organization of an Amazonian forest bird community. Ecological Monographs 60 (2): 213-238.

Viana, V.M.; A.A.J. Tabanez \& J.L.F. Batista. 1997. Dynamics and restoration of forest fragments in the Brazilian Atlantic Moist Forest, p. 351-365. In: W.F. LAURANCE \& R.O. BierRegaARd (Eds). Tropical forest remnants: ecology, management and conservation of fragmented communities. Chicago, University of Chicago Press, 632p.

WilLis, E.O. 1979. The composition of avian communities in remanescent woodlots in southern Brazil. Papéis Avulsos Zoologia 33 (1): 1-25.

Recebido em 11.VIII.2006; aceito em 14.XI.2007. 